\title{
Improved Discrete-Time Boundary Condition for the Thin-Wire FDTD Analysis of Lossy Insulated Cylindrical Antennas Located in Lossy Media
}

\author{
Seung-Yeup Hyun*
}

\begin{abstract}
For the thin-wire (TW) finite-difference time-domain (FDTD) analysis of lossy insulated antennas surrounded by lossy media, an improved discrete-time boundary condition (DTBC) at the interface is proposed here. In previous TW-FDTD techniques, the DTBC formulations on the material discontinuity between the lossy insulation and lossy surrounding media were derived from the dielectric constitutive relation under the uniform field approximation (UFA) over each time step. In this paper, to achieve higher accuracy, an improved DTBC is formulated from Maxwell's equations under the linear field approximation (LFA) and subsequently corrected in the TWFDTD update equation. By comparing the input impedances of Teflon-insulated cylindrical monopole antennas located in wet soils, we show that the proposed approach provides higher accuracy than previous techniques.
\end{abstract}

Key Words: Discrete-Time Boundary Condition, Finite-Difference Time-Domain Methods, Insulated Cylinders, Thin Wires.

\section{INTRODUCTION}

In telecommunications, geophysical explorations, and diagnostics, insulated cylindrical antennas have often been located in lossy media such as soil, water, or biological tissue [1]. The electromagnetic characteristics of antennas and wave propagation in inhomogeneous media have been analyzed using the finitedifference time-domain (FDTD) method [1-4]. However, since the standard FDTD is based on the time and space discretization of Maxwell's equations, the fine geometries of the antennas should be modeled as fine grids with sufficiently small cell sizes relative to the minimum wavelength. This results in low computational efficiency due to the large number of cells. One the other hand, thin-wire FDTD (TW-FDTD) treats a coarse grid with the pre-correction of near-field beha- vior around thin electrically insulated antennas $[3,4]$. Therefore, TW-FDTD can provide sufficient accuracy, equivalent to finegrid FDTD, while maintaining higher computational efficiency. In $[3,4]$, the loading effect of both the insulation and surrounding media was considered by using discrete-time boundary condition (DTBC) formulations. These were derived from the dielectric constitutive relation on the material interface between the lossy media under the uniform field approximation (UFA) over each time step. However, the electromagnetic fields on the material interface are fast varying, and the standard FDTD method is based on the linear field approximation (LFA) in time and space. Therefore, the LFA over each time interval may be suitable for the DTBC formulation. In this paper, we propose an improved DTBC by using a discrete-time version of Maxwell's equations under the LFA. The DTBC is corrected in the

Manuscript received July 24, 2020 ; Revised September 15, 2020 ; Accepted October 12, 2020. (ID No. 20200724-108J)

Department of Telecommunication Engineering, School of Electrical, Electronic, Telecommunication and Computer Engineering, Jeju National University, Jeju, Korea. "Corresponding Author: Seung-Yeup Hyun (e-mail: syhyun@jejunu.ac.kr)

This is an Open-Access article distributed under the terms of the Creative Commons Attribution Non-Commercial License (http://creativecommons.org/licenses/by-nc/4.0) which permits unrestricted non-commercial use, distribution, and reproduction in any medium, provided the original work is properly cited.

(c) Copyright The Korean Institute of Electromagnetic Engineering and Science. All Rights Reserved. 
TW-FDTD update equation. By comparing the input impedances of Teflon-insulated cylindrical monopole antennas located in wet soil, we show that this approach is more accurate than previous techniques.

\section{IMPROVED DTBC FORMULATION FOR TW-FDTD}

Fig. 1 shows a FDTD unit cell for the TW modeling of a lossy insulated cylinder in a lossy surrounding medium. A conductive cylinder of outer radius $a$ lies along the $z$-axis and is insulated with a lossy dielectric material of outer radius $\rho_{i}$, permittivity $\varepsilon_{i}$, and conductivity $\sigma_{i}$. The insulated cylinder is surrounded by a lossy dielectric medium of permittivity $\varepsilon_{s}$ and conductivity $\sigma_{s}$. Since the geometry of the cylinder is rotationally symmetric along the $\phi$-direction, we use the twodimensional (2D) cylindrical FDTD of the discrete space $(\rho, z)=(i \Delta \rho, j \Delta z) \equiv(i, j)$ and discrete time $t=n \Delta t \equiv n[4]$. Here, $\Delta \rho$ and $\Delta z$ are the spatial steps along the $\rho$ and $z$ axes, respectively, and $\Delta t$ is the time step. The variables $(i, j)$ and $n$ are the integer indices of the space and time discretization, respectively.

The LFA over each time step can be represented as the cen-

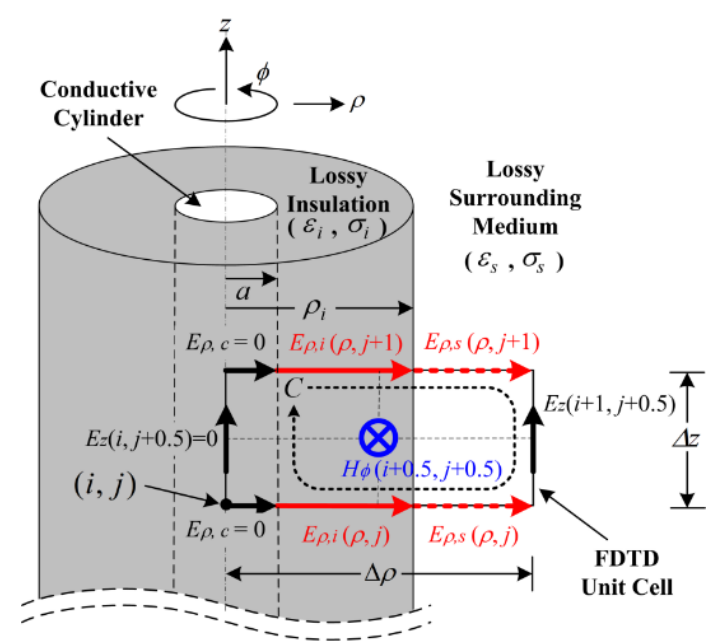

Fig. 1. TW-FDTD modeling of a lossy insulated cylinder surrounded by a lossy medium. tral-difference and the time-averaging schemes in the differential form of Ampere-Maxwell's law at $t=(n-0.5) \Delta t$ as

$$
\begin{aligned}
& \nabla \times \vec{H}^{n-0.5}(\rho, z)=\varepsilon \frac{\partial \vec{E}^{n-0.5}(\rho, z)}{\partial t}+\sigma \vec{E}^{n-0.5}(\rho, z) \\
& \approx \varepsilon\left[\frac{\vec{E}^{n}(\rho, z)-\vec{E}^{n-1}(\rho, z)}{\Delta t}\right]+\sigma\left[\frac{\vec{E}^{n}(\rho, z)+\vec{E}^{n-1}(\rho, z)}{2}\right] \\
& =\left(\frac{\varepsilon}{\Delta t}+\frac{\sigma}{2}\right) \vec{E}^{n}(\rho, z)-\left(\frac{\varepsilon}{\Delta t}-\frac{\sigma}{2}\right) \vec{E}^{n-1}(\rho, z) .
\end{aligned}
$$

In Fig. 1, the tangential electric and magnetic fields on the material interface $\left(\rho=\rho_{i}\right)$ between the insulating and the surrounding media should be continuous:

$$
\begin{aligned}
E_{z, i}^{n}\left(\rho_{i}, z\right) & =E_{z, s}^{n}\left(\rho_{i}, z\right) \\
H_{\phi, i}^{n-0.5}\left(\rho_{i}, z\right) & =H_{\phi, s}^{n-0.5}\left(\rho_{i}, z\right),
\end{aligned}
$$

where the subscripts $i$ and $s$ denote the insulating and surrounding media, respectively. Applying (2) and (3) to (1), an improved DTBC for the discontinuity of the normal electric fields on the interface can be obtained:

$$
\begin{aligned}
& \left(\frac{\varepsilon_{i}}{\Delta t}+\frac{\sigma_{i}}{2}\right) E_{\rho, i}^{n}\left(\rho_{i}, z\right)-\left(\frac{\varepsilon_{i}}{\Delta t}-\frac{\sigma_{i}}{2}\right) E_{\rho, i}^{n-1}\left(\rho_{i}, z\right) \\
= & \left(\frac{\varepsilon_{s}}{\Delta t}+\frac{\sigma_{s}}{2}\right) E_{\rho, s}^{n}\left(\rho_{i}, z\right)-\left(\frac{\varepsilon_{s}}{\Delta t}-\frac{\sigma_{s}}{2}\right) E_{\rho, s}^{n-1}\left(\rho_{i}, z\right) .
\end{aligned}
$$

From (4), the normal electric field at $t=n \Delta t$ in the surrounding medium on the interface can be given as

$$
\begin{gathered}
E_{\rho, s}^{n}\left(\rho_{i}, z\right)=K_{i s} E_{\rho, i}^{n}\left(\rho_{i}, z\right)+L_{i s} T_{s S}^{n}\left(\rho_{i}, z\right) \\
T_{s S}^{n}\left(\rho_{i}, z\right)=\sum_{m=1}^{n-1} M_{s s}{ }^{m-1} E_{\rho, i}^{n-m}\left(\rho_{i}, z\right) \\
K_{i s}=\frac{\varepsilon_{i}+\sigma_{i} \Delta t / 2}{\varepsilon_{s}+\sigma_{s} \Delta t / 2} \\
L_{i s}=\frac{-\left(\varepsilon_{i}-K_{i s} \varepsilon_{s}\right)+\left(\sigma_{i}-K_{i s} \sigma_{s}\right) \Delta t / 2}{\varepsilon_{s}+\sigma_{s} \Delta t / 2} \\
M_{S S}=\frac{\varepsilon_{s}-\sigma_{s} \Delta t / 2}{\varepsilon_{s}+\sigma_{s} \Delta t / 2} .
\end{gathered}
$$

In (5), the first term on the right-hand side is the DTBC of the present time $t=n \Delta t$, and the second term is the DTBC that includes the convolution effects of past times $t=$ $(n-1) \Delta t, \ldots, \Delta t$, and 0 . Table 1 shows a comparison of the DTBC coefficients used in previous versions of the technique and the proposed TW-FDTD framework. All TW-FDTD

Table 1. Comparison of the DTBC coefficients in different TW-FDTD formulations for lossy insulated cylinders in lossy surrounding media

\begin{tabular}{cccc}
\hline DTBC coefficients in (5) & Previous TW-FDTD [3] & Previous TW-FDTD [4] & Proposed TW-FDTD \\
\hline$K_{i s}$ & $\frac{\varepsilon_{i}+\sigma_{i} \Delta t / 2}{\varepsilon_{s}+\sigma_{s} \Delta t / 2}$ & $\frac{\varepsilon_{i}+\sigma_{i} \Delta t / 2}{\varepsilon_{s}+\sigma_{s} \Delta t / 2}$ & $\frac{\varepsilon_{i}+\sigma_{i} \Delta t / 2}{\varepsilon_{s}+\sigma_{s} \Delta t / 2}$ \\
$L_{i s}$ & 0 & $\frac{\left(\sigma_{i}-K_{i s} \sigma_{s}\right) \Delta t / 2}{\varepsilon_{s}+\sigma_{s} \Delta t / 2}$ & $\frac{-\left(\varepsilon_{i}-K_{i s} \varepsilon_{s}\right)+\left(\sigma_{i}-K_{i s} \sigma_{s}\right) \Delta t / 2}{\varepsilon_{s}+\sigma_{s} \Delta t / 2}$ \\
$M_{s s}$ & 0 & $\frac{\varepsilon_{s}}{\varepsilon_{s}+\sigma_{s} \Delta t / 2}$ & $\frac{\varepsilon_{s}-\sigma_{s} \Delta t / 2}{\varepsilon_{s}+\sigma_{s} \Delta t / 2}$ \\
\hline
\end{tabular}


equations, except for those for the DTBC coefficients, are the same as those in [4]. Therefore, they were omitted here for brevity.

\section{COMPARISON RESULTS}

To evaluate the computational accuracy of the proposed TWFDTD technique using the improved DTBC formulation, we calculate the input impedances $\left(Z_{\text {in }}=R_{\text {in }}+j X_{\text {in }}\right)$ of Tefloninsulated cylindrical monopole antennas in wet soils. The conductive cylinder of an antenna has $a=0.635 \mathrm{~mm}$, an axial length of $100 a$, and $\rho_{i}=1.614 a$ and is insulated with Teflon $\left(\varepsilon_{i}=2.1 \varepsilon_{0}, \sigma_{i}=5.0 \mu \mathrm{S} / \mathrm{m}\right.$ [4]). The insulated antennas are surrounded by $5 \%-25 \%$ wet soil and are fed by a $50 \Omega$ coaxial line. In all FDTD simulations, the spatial steps along the radial and axial directions are $\Delta \rho=3.23 a$ and $\Delta z=a$, respectively. Under the stability condition, we use a time step of $\Delta t=$ $\min (\Delta \rho, \Delta z) / 2 v$, where $v$ is the wave velocity in the medium. First, in the case of the Teflon-insulated monopole antenna inserted in $15 \%$ wet soil $\left(\varepsilon_{s}=5.3 \varepsilon_{0}\right.$ and $\sigma_{s}=0.117 \mathrm{~S} / \mathrm{m} \mathrm{[5]),}$ the results of our approach are compared with those of previous TW-FDTD formulations [3, 4] and with fine-grid finiteelement method (FEM) data, as shown in Fig. 2. It can be seen that the results of previous TW-FDTD models show some errors compared with the FEM data. This is because [3] and [4] are based on the first-term DTBC and full-term DTBC under the UFA over each time step, respectively. Since the proposed TW-FDTD technique uses the full-term DTBC under the LFA over each time interval, it provides high accuracy, comparable with the FEM data. Next, the effect of the wet soil as a function of the volumetric moisture content is investigated, as shown in Fig. 3. We calculate the input impedances of the Teflon-insulated monopole antennas inserted in 5\%-25\% wet soils $\left(\varepsilon_{s}=2.8 \varepsilon_{0}-10.2 \varepsilon_{0}\right.$ and $\left.\sigma_{s}=0.039-0.218 \mathrm{~S} / \mathrm{m}[5]\right)$. One can observe that the results of the proposed TW- FDTD model are in good agreement with the FEM data.

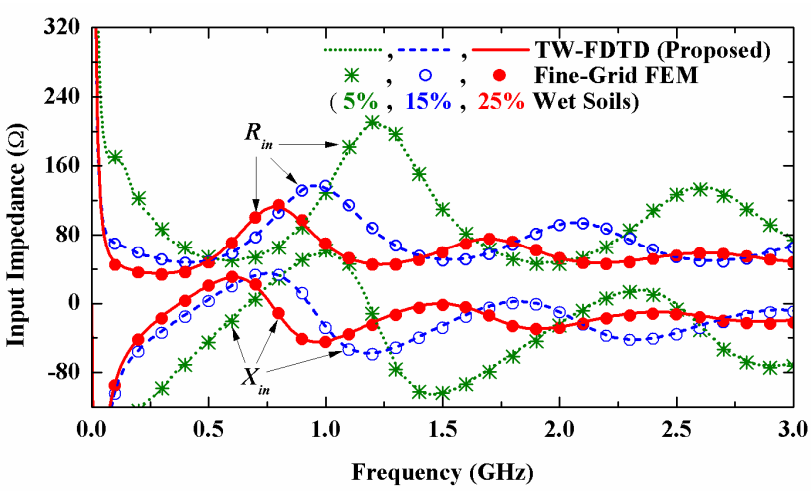

Fig. 3. The input impedances of Teflon-insulated cylindrical monopole antennas embedded in 5\%-25\% wet soils.

\section{CONCLUSION}

An improved DTBC formulation for the TW-FDTD modeling of insulated antennas located in lossy media is proposed. The improved DTBC between the insulation and surrounding media was derived from Maxwell's equations under the LFA over each time step. Then, the DTBC was corrected in the TW-FDTD equations. In a comparative study of the input impedances of Teflon-insulated monopole antennas in wet soils, we show that the proposed approach has higher accuracy than previous techniques.

This research was supported by the 2019 scientific promotion program funded by Jeju National University.

\section{REFERENCES}

[1] T. W. Hertel and G. S. Smith, "The insulated linear antenna-revisited," IEEE Transactions on Antennas and Propagation, vol. 48, no. 6, pp. 914-920, 2000.

[2] J. H. Kweon, M. S. Park, J. H. Cho, and K. Y.Jung, "FDTD analysis of electromagnetic wave propagation in an inho

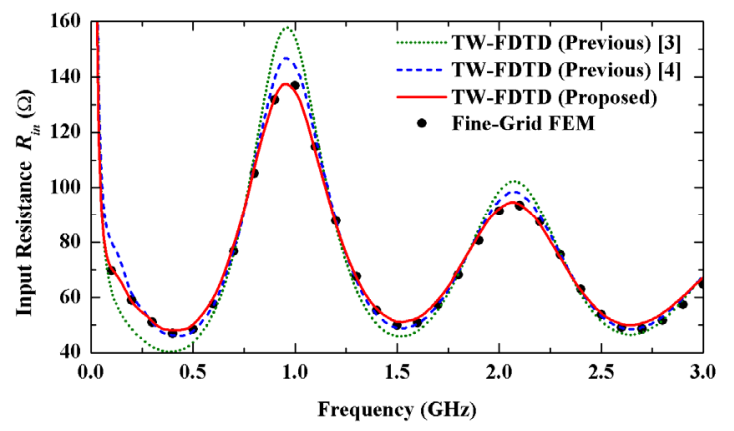

(a)

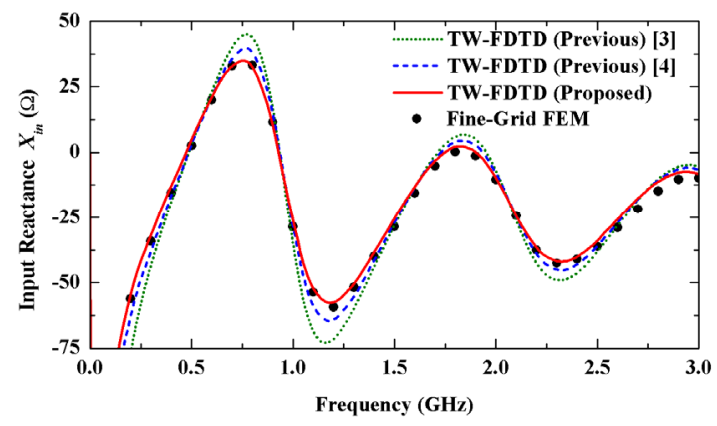

(b)

Fig. 2. The input impedances of Teflon-insulated cylindrical monopole antennas placed in $15 \%$ wet soil: (a) input resistance and (b) input reactance. 
mogeneous ionosphere under arbitrary-direction geomagnetic field," Journal of Electromagnetic Engineering and Science, vol. 18, no. 3, pp. 212-214, 2018.

[3] J. J. Boonzaaier and C. W. I. Pistonius, "Finite-difference time-domain field approximations for thin wires with a lossy coating," IEE Proceedings-Microwave, Antennas \& Propagation, vol. 141, no. 2, pp. 107-113, 1994.

[4] S. Y. Hyun and S. Y. Kim, "Thin-wire model using subcellular extensions in the finite-difference time-domain

\section{Seung-Yeup Hyun}

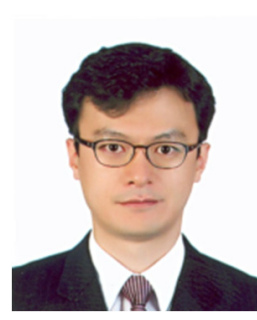

received the B.S. degree in telecommunications engineering from Jeju National University, Jeju, Korea, in 1997, and the M.S. and Ph.D. degrees in radio sciences and engineering from Korea University, Seoul, Korea, in 1999 and 2008, respectively. From 2001 to 2003, he was a Staff Research Engineer with the Research and Development Laboratory, SG Technology, Chungnam, Korea. From 2003 to 2006, he was a Senior Research Engineer with the Digital Appliance Laboratory, LG Electronics, Seoul, Korea. From 1997 to 2008, he was a Student Researcher with the Imaging Media Research Center, Korea Institute of Science and Technology (KIST), Seoul, Korea. From 2009 to 2011, he was a Principal Research Engineer with the Ground-Penetrating Radar (GPR) Division, Isung Corporation, Gyeonggi-do, Korea. From 2008 to 2012, he was a Postdoctoral Research Fellow with the Imaging Media Research Center, KIST. Since 2012, he has been a faculty member of the Department of Telecommunication Engineering, Jeju National University, Jeju, Korea, where he is currently an associate professor. His research interests include ultra-wideband (UWB) antennas, microwave imaging, groundpenetrating radar (GPR) systems, and plasma lighting systems. analysis of thin and lossy insulated cylindrical structures in lossy media," IEEE Transactions on Electromagnetic Compatibility, vol. 51, no. 4, pp. 1009-1016, 2009.

[5] M. T. Hallikainen, F. T. Ulaby, M. C. Dobson, M. A. ElRayes, and L. K. Wu, "Microwave dielectric behavior of wet soil, Part I: Empirical models and experimental observations," IEEE Transactions on Geoscience and Remote Sensing, vol. 23, no. 1, pp. 25-34, 1985. 